\title{
Sonochemical Reactor Treating Drinking Water for Pigs
}

\author{
S. Shestakov ${ }^{1}$, O. Krasulya ${ }^{1}$, T. Shlenskaya ${ }^{1}$, T. Baulina ${ }^{1}$, E. Smeshek ${ }^{2}$ \\ ${ }^{1}$ Moscow State University of Technology and Management, Russia \\ ${ }^{2}$ Polessky state university, Republic of Belarus
}

\begin{abstract}
The article deals with the use of activated water for drinking of pigs. Moreover, is described a method sonochemical activation of water. Sonochemical activated water is devoid of own supramolecular structure and has a high solvent power similar to how a boiling water, while remaining virtually the same temperature, which was prior to treatment. It is shown that the water in a thermodynamically nonequilibrium state, in which abideth some time as a result of such treatment, and has bactericidal and bacteriostatic properties, which it retains long time. All this suggests that the hydration of biopolymers directly into the esophagus and stomach of animals during their drinking will give tangible effect. Given the description of the mechanisms of cavitational disintegrated of water and described in comparison reactors for water treatment. Said that a regulatory authority in Russia give permission to use sonochemical technologies and devices in the food industry. Described sonochemical reactor, designed specifically for use in drinking of pigs, which have a reduced noise level and power consumption, but with better performance.
\end{abstract}

Keywords: - hydration and structuration of biopolymers, non-stationary thermodynamically state of the water, sonochemistry, sonochemical cavitation reactor.

\section{INTRODUCTION}

Water plays an important role when the fattening pigs. Water used for both sanitary and technological needs, and directly for drinking pigs. Plentiful drink need especially when fatten with dry foods [1,2]. As equipment are use nipple drinkers. In fattening complexes where there are sources of groundwater, they are attached to the network of water tower or directly to the network of installation for lifting water from wells. In the period of snow break or heavy precipitation as rain contaminated surface water can get into the water fed to animals. [3] Use of contaminated water can lead to the spread of infectious diseases among animals. Therefore, a special water treatment before its supply from the mains to the animals is need.

The use of activated in different ways drinking water for animals is well known. Activation are doing not only for the water treatment and disinfection, but also for increase the weight gain of the animals $[3,4]$. In conducting joint Russian-Belarusian Studies in Food sonochemistry [5], there was an interesting idea to apply sonochemical activated water for intensive hydration of biopolymers pigs feeds. It has been hypothesized that it could be applied when pigs has drinking. Then hydration will have place directly into the esophagus and stomach of animals. Prepared for this water should be supplied in drinking bowls for pigs which, for example, were designed and manufactured in the Republic of Belarus company "Demeter". It was decided to equip them with special sonochemical reactors. The combination of feeding dry food with watering of sonochemical treated water will allow to the pigs better absorb food and increase weight gain. Thus, in the article [3] at the daily consumption of treated water cavitation larger than usual, weight gain increased by $6.5 \%$. In this case, the consumption of specific energy for water treatment amounted to $34.4 \mathrm{~kW} \cdot \mathrm{h} / \mathrm{t}$. The idea was supported by the Academician P. Vityaz of National Academy of Sciences. Under his leadership, the regional office of the Russian Acoustical Society developed a special sonochemical reactor [6].

\section{SUBJECT OF SONOCHEMISTRY}

Sonochemistry are high-energy chemistry. Nowadays, regarding sonochemistry, including the food processing industry and in agriculture, is held active research worldwide. Studies have begun and in the Moscow State University of Technology and Management (MSUTM) after the organization of it in 2009, the laboratory of food sonochemistry. This allowed him to stand on a par with the world's leading educational institutions - universities, Coventry, Illinois, Hohenheim, Melbourne and Mexico City. Was performed a sanitary-epidemiological examination and certification of designed in university the cavitation reactors by TU 5130-002-26784341-08 for implement of them into the sonochemical technology of food inductry [7]. Federal Service for Supervision of Consumer Rights Protection and Human Welfare and State Standard of Russian the reactors are allowed to produce for use in the technological devices of food industry. These reactors (Fig. 1) allow us to replenish incurred of moisture losses on the stages of storage and transport of food raw material particularly 
in the dried and froze. It is believed that the pure protein can theoretically bind in the hydration reaction of up to $40 \%$ of the water of its weight. [8].

The main factor of the sonochemical reactions is a cavitation in the liquid at which generates giant impulses of pressure generated by the cavitation bubbles. Since the total amount of contents of bubbles, compared to the volume of the liquid is negligible, so that sonochemical reactions is occurring in the gas phase inside the bubbles have little practical importance. Practically significant sonochemical reactions is occur in the liquid phase as a result of distributing therein of the pressure pulses from the bubbles, stimulating the reaction of solutes through a mechanical impact on the structure of their hydrate shell and the most water. The thermal dissociation of water which going in the gas phase bubbles results in only a slight shift of $p H$ to the alkaline region, and the synthesis of hydrogen peroxide in amounts of thousandths units on the weight of water. Therefore, water for drinking of pigs not necessary to protect from education in her structure radicals and peroxide compounds, as is done in the food sonochemistry, since they are bactericidal agents. This makes it possible exception of chlorine, which commonly used to disinfect water. Food safety sonochemical water treatment has been studied experimentally. This was determined by biotesting by methodics the toxicological assessment of food products [9]. Was compared the number of ciliates Tetrahymena pyriformis in samples of spray drying serum which was restored on sonochemical treated water at regular intervals. In the serum of restored at the water which was subjected cavitation process throughout the experiment the number of ciliates was higher than in the serum, the recovered on normal water. And, moreover, the increase amount of their with time there prevail also. This is because the cavitation treatment significantly alters the physicochemical properties of the water and not creates the factors adversely affecting the growth and reproduction of protozoa. The whey the better soluble in such water and the nutritional value of the solution turns above. It also emerged that arise and is good saved the bacteriostatic properties of the treated water.

\begin{tabular}{|l|c|c|}
\hline \multicolumn{1}{|c|}{$\begin{array}{c}\text { Time after } \\
5 \text { min treatment with }\end{array}$} & $\begin{array}{c}\text { Absolute } \\
\text { viscosity, } c P s\end{array}$ & $\begin{array}{c}\text { Viable microorganisms } \\
\text { by the test [10] }\end{array}$ \\
\hline Two hours & $0.971 \pm 0.008$ & 0.630 \\
24 hours & $0.987 \pm 0.004$ & 0.849 \\
\hline Control of & $1.019 \pm 0.007$ & 1.760 \\
\hline
\end{tabular}

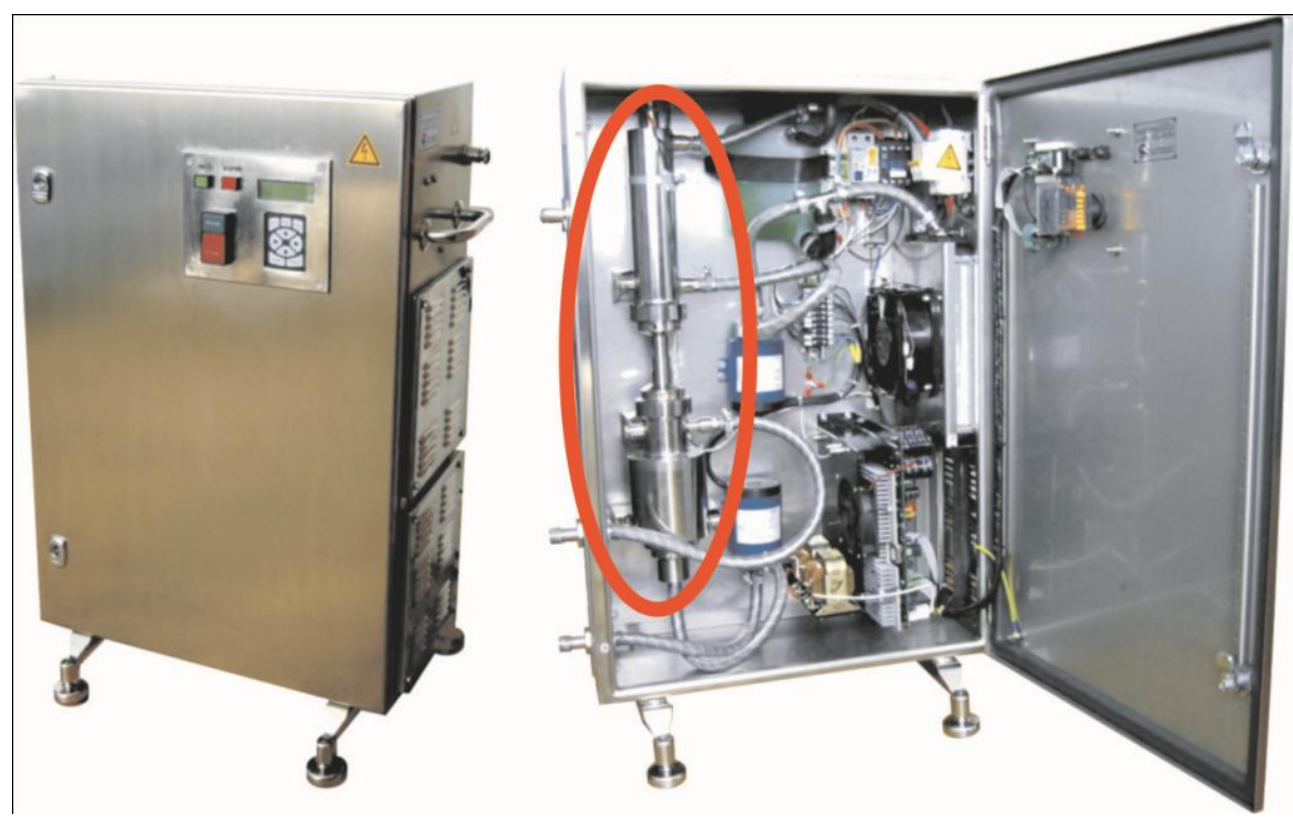

Fig. 1. Exterior and interior view installation sonochemical processing of salt brines for the cooking of meat food products with the reactor by TU 5130-002-26784341-08 (circled in red).

The transformation of the energy of pressure pulses of the cavitation in the water epithermal implements the mechanism of destruction of hydration shells of ions and colloids dissolved substances that prevent their entry into chemical reactions. Therefore cavitation easily turn a soluble calcium bicarbonate $\mathrm{Ca}\left(\mathrm{HCO}_{3}\right)_{2}$ and $\mathrm{Mg}\left(\mathrm{HCO}_{3}\right)_{2}$ into form insoluble amorphous colloidal $\mathrm{CaCO}_{3}$, and $\mathrm{MgCO}_{3}$, what doing softening water [11]. Water even at room temperature has an associative supramolecular structure [12] (Fig. 2). 


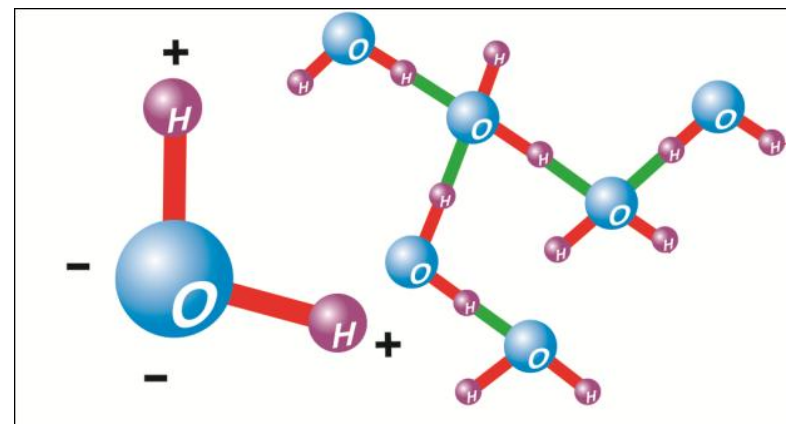

Fig. 2. The structure of water molecules (left) and a fragment of an associative water structure (right). Covalent bonds are marked with red and hydrogen bonds - green.

Under the influence of cavitation pulses pressure the water on time acquires thermodynamically nonequilibrium state, which is characterized by its anomalously high solvent ability (Fig. 3). It lasts until the received energy gradually will be returned in the form of heat of hydration, which occurs between the water molecules, again restoring the hydrogen bond and structure of water that correspond to thermodynamic equilibrium. That is, over time, inevitably occurs the relaxation of the non-equilibrium state.

$$
\mathrm{H}_{2} \mathrm{O}+\mathrm{H}_{2} \mathrm{O}+\ldots+\mathrm{H}_{2} \mathrm{O} \leftrightarrow m\left(\mathrm{H}_{2} \mathrm{O}\right)_{n}+25 \frac{n \cdot m}{\mathrm{~N}_{A}} \mathrm{~kJ}
$$

where: $m$ - the number of molecular water associates that react; $n$ - number of water molecules that consist in an associating; $\mathrm{N}_{\mathrm{A}}-$ Avogadro's number.

If immediately after the cavitation in the water dissolve the dry biomass, then dissolved in it electrolytes almost completely dissociated on the ions which will be immobilized of the water monomolecules and the protein molecules acquire dense hydrated shells. This will increase the mass of the protein, since the water connect to it through the mechanisms similar to those that occur in nature in the process of it synthesis and almost also strong, how a ties which form a natural protein structure.

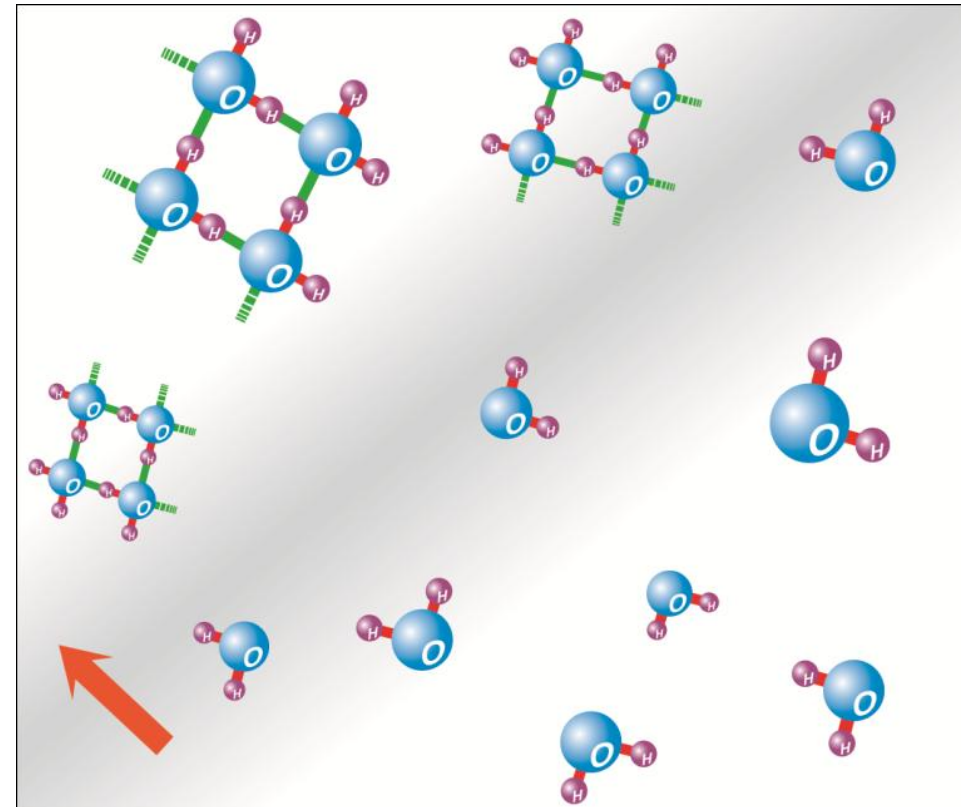

Fig. 3. The destruction of the molecular associates of water by pressure pulse from the cavitation bubble. The arrow indicates the direction move of the pulse.

In addition, may arising, the quaternary structure of the protein (Fig. 4) formed by the so-called the structuring by hydration [13].

But the energy of cavitation is the cause of erosional destruction of solid materials [13]. Erosion may be subject and the materials used for the components of the cavitations reactors design - the enclosure and acoustic cell. Products erosion, falling into the treated water, can irreversibly change its physical and chemical properties, what is strictly not allowed for treating of drinking water. 


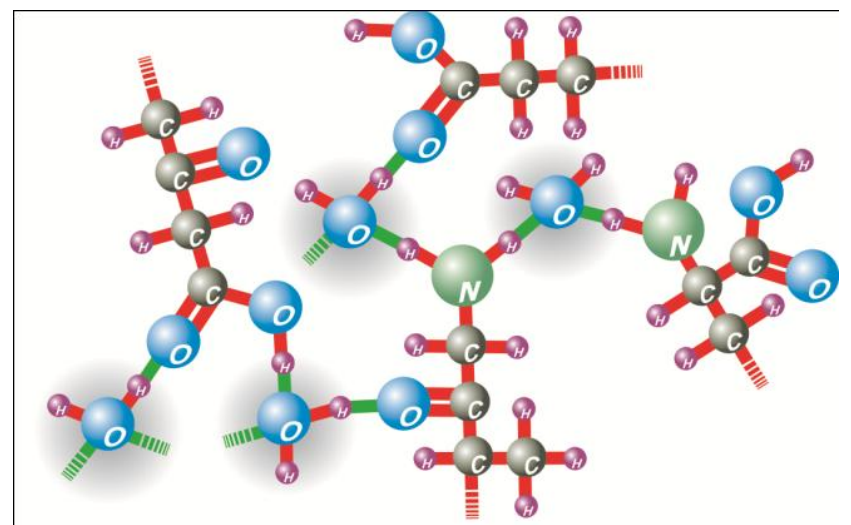

Fig. 4. Hydration and structuring of protein by hydration. Water molecules which are hydrated of the active centers of aminoacids of the protein are shaded.

\section{INFORMATION ABOUT REACTORS FOR WATER TREATING}

This deficiency is not in the reactor, specifically designed for watering pigs [3], where acoustic cell has two opposite and working in one phase a membrane transducers. That is them radiating surfaces doing into liquid the fluctuation in opposite directions (Fig. 5).

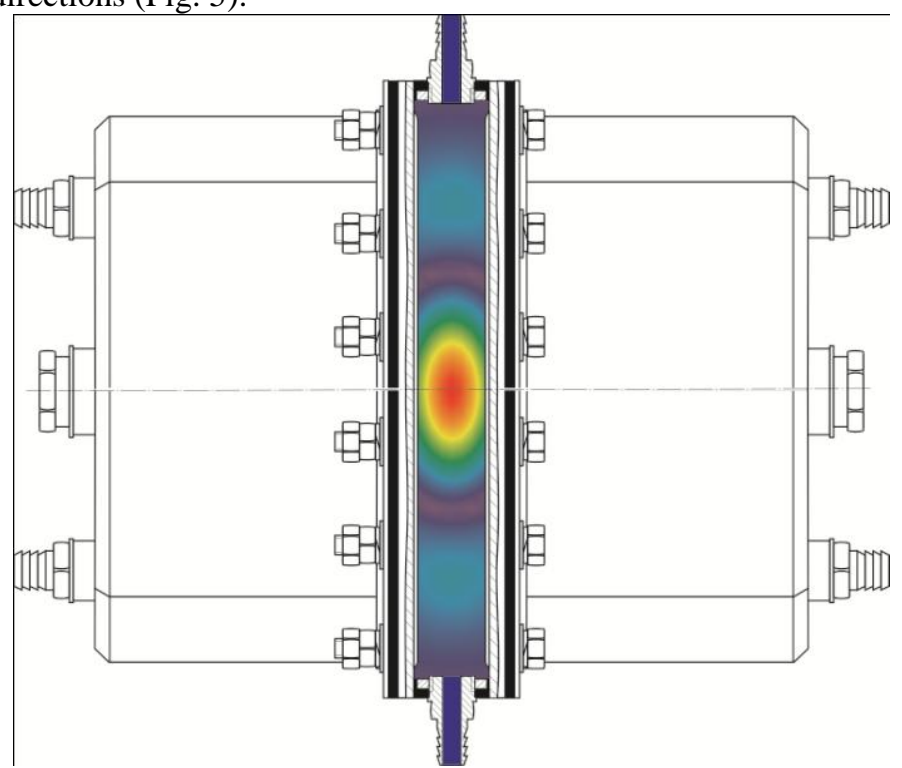

Fig. 5. Design of reactor from [3]. In the plane of the section the tonal pattern shows a distribution of the bulk density of the erosive power of cavitation.

They are spaced apart by one-half wavelength of ultrasound in water, and the side wall of the housing is located near the node of the flexural vibration of the radiating membrane. Therefore, its erosion is negligible. Such a reactor has a large volume of the working chamber, but its specific productivity is not very high. In it used a magnetostrictive method of electroacoustic conversion with efficiency about $50 \%$. In addition, he has a big noise emission, as magnetostrictive transducers are cooled with water, in which also acts cavitation, which is not desirable for pigs [14]. It is installed as stated in its description on the water tank for 25 pigs. And when comes the maximum daily water consumption it will provide only half of the water needs, even if it is included in the recirculation circuit of storage tank.

IV.

HYPOTHESIS

If considering the volume of the liquid in the reactor, enclosed between the planes of the solid-state resonator, from of which occurs spread fluctuations, as part of the vibration system of acoustic reactor cell, then you can create the optimum conditions for the superposition of these fluctuations. Under such conditions the areas of cavitation, which are formed near the antinodes of the pressure resulting wave, will occupy the largest volume [7], what will make the reactor more productive. This can be done, given the well-known fact that the elastic plane wave in water is capable of produce the cavitation energy at a distance of no more than three half- 
waves, as well as that on distance of length of the half-wave oscillations in the metals may take place about a three half-wave oscillations of the same frequency in the liquid. That is, the working length of acoustic cell of the reactor should be equal to 1.5 wavelength of the elastic oscillations of the liquid when the radiating surfaces are spaced apart on 0.5 oscillation wavelength of the metal. Then height of acoustic cell must be exactly equal to half the oscillation wavelength of the metal at the same frequency. Such conditions are easier to perform, when the solid-state part of the vibrating system acoustic cell is symmetrical about the center of mass, i.e., when the transducers are located on both sides of the treated water. In addition, in geometrically symmetrical about its center of mass system of elastic waves would be better hold resonance. Cavitation erosion radiating surfaces wherein are practically not. The surface of the reactor vessel, optimally choosing its diameter, can be placed in the erosion-safe area. A interconnecting the two emitting surface of a solid-state resonator element (jumper), you can protect against cavitation thin erosion-resistants coated, such as rubber or plastic. The effect of this decision is to increase the dissipated power in the acoustic cavitation and increasing processing performance without increasing the erosion of its surfaces and without increasing the intensity of the external noise.

\section{THEORY AND COMPUTER EXPERIMENT}

A comparison was made such reactor with the reactor from [3]. The comparison was performed by computer simulation with via mathematical model of the cavitation reactor and the similarity principle cavitational processes [15]. As a reference, was selected the reactor from [16] (Fig. 6).

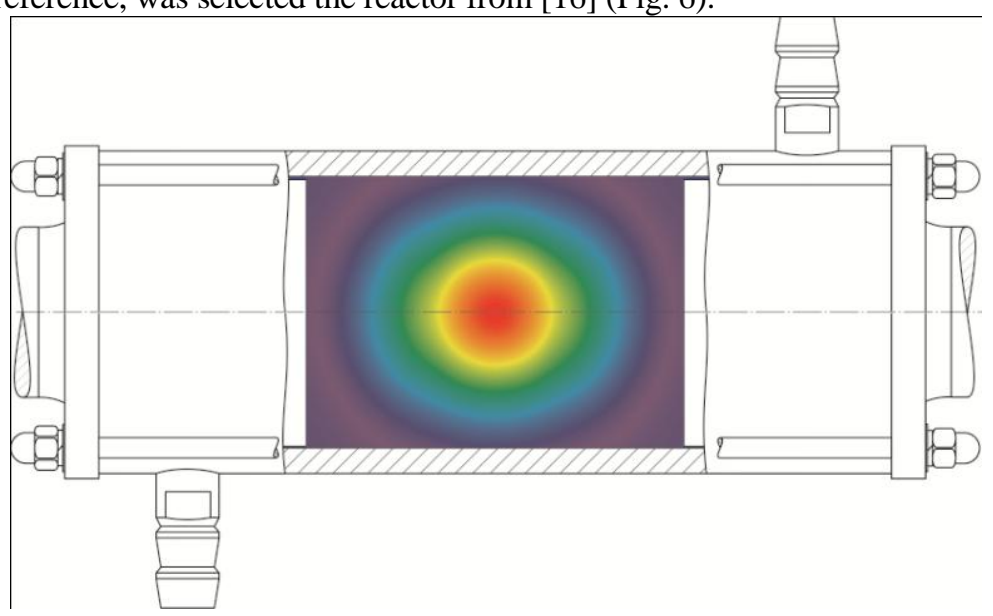

Fig. 6. The design of the reactor from [16]

It consists of a camera body of the seamless stainless steel pipe. Acoustical transformers, which transmit vibrations into the water from piezoelectric transducer electrical output of $1.2 \mathrm{~kW}$ secured therein by the flanges bolts and nuts. Through the working volume of the reactor water passes through the nozzles.

Designed reactor (Fig. 7) consists of a camera body with flanges.

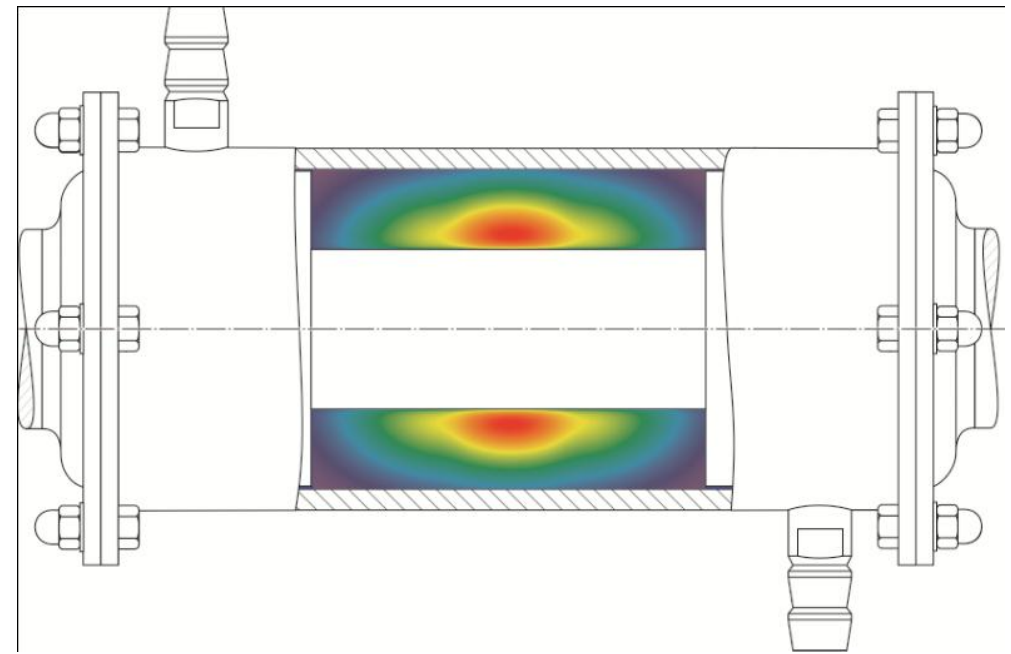

Fig. 7. The design of the developed reactor with a monolithic solid-state vibrational system of the resonance cell. 
In it using bolts and nuts fastened acoustic waveguide transformers which transmit vibrations of piezoceramic transducer electrical output of $1.2 \mathrm{~kW}$ into the water. Both transformers are interconnected into the single solid-state symmetrical vibrational system. A source power fluctuation, the maximum pressure amplitude total wave is the same as that of the reactor from [16].

When compared was modeled of the performance of the reactor, which was calculated as the product of the integral in the working volume of reactor $V$ the density of the erosive power of cavitation:

$$
\bar{Q}=f \iiint_{(V)} a^{2} \Sigma^{2} v_{i} \frac{\{\tau+\Delta \tau\}-\{\tau+\Delta \tau\}^{2}}{(\tau+\Delta \tau)^{2}} \mathrm{~d} x \mathrm{~d} y \mathrm{~d} \alpha,
$$

where: $f$ - oscillation frequency; $a$ - average attenuation coefficient of the total pressure perturbation from the all bubbles in the design point; $\Sigma v_{i}=S \cdot \Sigma h_{i}, i=0 \ldots n$ - total volume of cavitation fields $(S$ - area of the radiating surface fluctuations); $\tau$ - the average dimensionless time the arrival of pressure disturbances from all the bubbles in the design point; $\Delta \tau=\frac{1}{2 n}\left[\frac{n}{2}\right]-$ an amendment on phase of the cavitation area [15]; in square brackets whole part, and in curly braces - the fractional part of the number.

The mean mileage of the pressure perturbation of cavitation in the period of the harmonic wave is set equal to the length of this wave $\lambda$. Dimensions $h_{i}$ cavitation regions along the line of the waves were calculated in angular units of phase as the difference between the values of the even and odd positive roots of the transcendental equation:

$$
A_{\max }|\sin \phi| \cdot\left[(1-\sqrt{\eta})^{2 \frac{\phi}{\pi}}+(1-\sqrt{\eta})^{2 n-2 \frac{\phi}{\pi}}\right]-A_{0}=0
$$

where $A_{\max }, A_{0}$ - the maximum at the emission of vibrations in the half-space of the liquid and threshold of cavitation for the amplitude of the sound pressure of wave; $\eta$ - cavitational scattering coefficient of wave energy. In the linear units they are transformed by means of dividing at the wave number $2 \pi / \lambda$. Erosion (pollution of water by erosion products) was calculated how the integral value of the bulk density of the erosive power in water near the metal surfaces of the reactor. The comparison in the values relative to the reactor on Fig. 6 shown below:

\begin{tabular}{|l|c|c|c|}
\hline \multicolumn{1}{|r|}{ REACTOR INDEX } & Fig.5 & Fig. 6 & Fig. 7 \\
\hline The electric power, $k W$ & 8,0 & 2,4 & 2,4 \\
Performance of water & 0,4 & 0,4 & 0,9 \\
treatment, $m^{3} / h$ & & & \\
Relative erosion, rel.u & 2,7 & 1 & 2,2 \\
\hline
\end{tabular}

\section{DISCUSSION OF EXPERIMENT AND CONCLUSIONS}

The table shows that the reactor in Fig. 5 has in 2.25 times performance of water treatment smaller than the reactor in Fig. 7 and higher erosion of construction details. It is understood that the reactor in Fig. 7, since the processing performance higher, that contaminating water by products erosion (by metal ions) is less than unity. This reactor at complex for fattening pigs can be included in the recirculation circuit of the storage tank for water.

\section{REFERENCES}

[1] S. Alexandrov, E. Prokopenko Guide to feeding pigs, Moscow, Donetsk: AST Stolker, 2004

[2] V. Kabanov Svinovodstvo, Moscow: Kolos, 2001

[3] N. Podkhomutov, V. Suchkov Water treatment using ultrasound for watering animals in the unit of pig fattening, Technology, equipment and components for the production of meat products a healthy diet: Proc. Intern. scientific-practical workshop, Vologda, "Gardening", 2004

[4] A. Apalikov Productive qualities of young pigs at using an activated water Dissertation PhD, Samara, 2004

[5] Shestakov S., Krasulya O., Smeshek E. Sonochemistry of food - area high-energy chemistry which actively is researched now in Russia and Belarus Materials IX International scientific-practical conference «Capabilities of modern science - 2013». Chemistry and Chemical technology Praha: Publishing House «Education and Science», 2013, pp 17-25

[6] Development of the technical proposal "A sonochemical reactor for the preparation of water at hydration of biopolymers dry feed pigs for their at watering" Report on experimental design work. Russian Acoustical Society. Vologda regional office, will teach. hands. P. Vityaz, performer E. Smeshek, State No 01201367171,2013

[7] S. Shestakov Food sonochemistry: the concept, the theoretical aspects and practical applications. Saarbruecken: LAMBERT Academic Publishing, 2012 
[8] The water in food products. Ed. R. Duckworth. Moscow: Food Industry, 1980

[9] E. Cheremnyh, E. Simbireva The ciliates are tasting to food. Chemistry and Life, 1, 2009

[10] A. Stekhin, G. Yakovleva A structured water. Nonlinear effects. Moscow: LCI Publisher, 2008

[11] N. Tikhomirov et al. A method of reducing temporary hardness of water in the stream and cavitation reactor for its implementation. Patent RU 2422371, C02F 1/36

[12] Jinesh K.B., Frenken J. W.M. Experimental evidence for ice formation at room temperature. Physical Review Letters, 101, 2008, 036101

[13] S. Shestakov The basic technology of cavitation disintegration. Moscow: EVA-Press, 2001

[14] N. Bazanova et al. Physiology of agricultural animals. Moscow: Kolos, 1980

[15] C. Shestakov Multibubble acoustic cavitation: A mathematical model and a physical similarity. Electronic Journal "Technical Acoustics», http://www.ejta.org, 2010, 14

[16] Development of the technical proposal "A cavitation reactor for the sonochemical water treatment and preparation of water and aqueous solutions for hydratation of various substances " Report on experimental design work. Russian Acoustical Society. Vologda regional office. State No 01201254896, 2012 